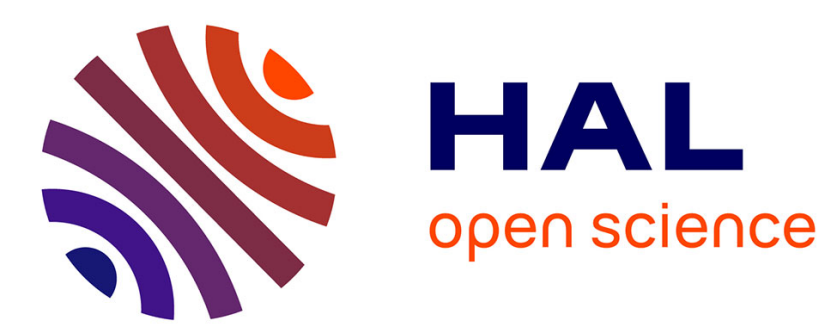

\title{
Autonomie ou dépendance sémantique des noms d'événements en français
}

Richard Huyghe

\section{To cite this version:}

Richard Huyghe. Autonomie ou dépendance sémantique des noms d'événements en français. Travaux de Linguistique: Revue Internationale de Linguistique Française, 2013, 67, pp.7-23. hal-00975573

\section{HAL Id: hal-00975573 \\ https://hal-univ-paris.archives-ouvertes.fr/hal-00975573}

Submitted on 9 Apr 2014

HAL is a multi-disciplinary open access archive for the deposit and dissemination of scientific research documents, whether they are published or not. The documents may come from teaching and research institutions in France or abroad, or from public or private research centers.
L'archive ouverte pluridisciplinaire $\mathbf{H A L}$, est destinée au dépôt et à la diffusion de documents scientifiques de niveau recherche, publiés ou non, émanant des établissements d'enseignement et de recherche français ou étrangers, des laboratoires publics ou privés. 
HuYGHE Richard. 2013. Autonomie ou dépendance sémantique des noms d'événements en français. Travaux de Linguistique 67, 7-23.

\title{
Autonomie ou dépendance sémantique des noms d'événements en français
}

\section{Résumé}

Parmi les noms traditionnellement considérés comme noms d'événements [Nev], i.e. pouvant servir de noyau à un $\mathrm{SN}$ sujet de avoir lieu, on distingue deux types de N. Les Nev autonomes (e.g. concert, avalanche) suffisent à la description d'événements, tandis que les Nev dépendants (e.g. augmentation, démantèlement) requièrent un complément pour identifier des événements. La distinction entre ces deux types de $\mathrm{N}$ n'équivaut pas à celle, proposée par Grimshaw (1990), entre « simple event nominals » et « complex event nominals ». Il est établi que la répartition entre $\mathrm{Nev}$ autonomes et dépendants ne repose pas sur des propriétés strictement morphologiques ou syntaxiques, et que la structure sémantique, i.e. l'ensemble des traits sémantiques pertinents, n'est pas la même pour tous les Nev. L'hypothèse est faite que l'autonomie ou la dépendance des Nev peut conditionner le type d'événement dénoté.

\section{The semantic autonomy or dependency of nouns denoting events in French}

\begin{abstract}
Two categories of nouns denoting events [ENs], i.e. of nouns which can be the subject of avoir lieu ('take place'), can be distinguished. Autonomous ENs (e.g. concert 'concert', avalanche 'avalanche') provide a sufficient description of events, whereas dependent ENs (e.g. augmentation 'increase', démantèlement 'dismantling'), need a complement to denote events. The distinction between these two categories is not equivalent to the one proposed by Grimshaw (1990), between " simple event nominals » and " complex event nominals ». It is shown that the distribution between autonomous and dependent ENs does not rely on pure syntactic or morphological properties, and that the semantic structure, i.e. the relevant semantic features, is not the same for all ENs. Furthermore, the autonomy or dependency of ENs may determine the ontological type of events denoted by ENs.
\end{abstract}




\title{
Autonomie ou dépendance sémantique des noms d'événements en français
}

\author{
Richard Huyghe \\ Université Paris Diderot, EA 3967 CLILLAC-ARP \\ rhuyghe@eila.univ-paris-diderot.fr
}

Les travaux sur la sémantique des noms d'événements [Nev] permettent à la fois de distinguer ces noms des catégories nominales voisines et de faire apparaître différents types de Nev. Nous revenons dans cet article sur l'hétérogénéité des Nev en français, en nous attardant sur leur capacité à dénoter d'eux-mêmes des événements, suivant certaines de leurs propriétés syntactico-sémantiques.

Est considéré ici comme Nev tout N qui peut constituer la tête d'un SN sujet de avoir lieu (cf. Balibar-Mrabti 1990, Gaatone 1992, Gross \& Kiefer 1995, Godard \& Jayez 1996). Tel est le cas de concert, réunion, manifestation et festival dans (1):
a. Un concert a lieu dans le parc.
b. Une réunion des délégués du personnel a eu lieu début mars.
c. Une manifestation a eu lieu devant le ministère ce matin.
d. Un festival de théâtre de rue a lieu tous les ans à Chalon-sur-Saône.

Les Nev dénotent des entités dynamiques et occurrentielles, i.e. dont l'existence implique l'ancrage et la délimitation temporels. Les Nev se distinguent ainsi :

- des noms d'objets [Nobj], comme arbre, chaise et maison, qui ne dénotent pas des entités dépendant directement du temps (cf. Godard \& Jayez 1996, Huyghe 2011 et 2012a),

- des noms de propriétés, comme intelligence, tristesse et banalité, qui ne dénotent pas des situations dynamiques (cf. Flaux \& Van de Velde 2000, Koehl 2009, Dal \& Namer 2010, Martin à paraître),

- des noms d'activités, comme jardinage, natation et braconnage, qui par leur caractère massif ne dénotent pas des actions délimitées dans le temps (cf. Flaux \& Van de Velde 2000, Heyd \& Knittel 2009, Haas \& Huyghe 2010),

- des noms d'« idéalités » (cf. Flaux \& Stosic 2011) comme chanson, film et symphonie, parfois appelés « noms d'événements faibles » (cf. Godard \& Jayez 1996), mais dont le caractère événementiel est discuté, précisément parce qu'ils ne dénotent pas des occurrences temporelles (i.e. des entités identifiées par leur localisation dans le temps $)^{1}$.

Une caractéristique essentielle des Nev est qu'ils décrivent des entités qui s'inscrivent dans le temps. Les Nev sont de fait compatibles avec les expressions de localisation temporelle. Ils peuvent, d'une part, dénoter une cible temporelle (i.e. une entité localisée dans le temps), dans des phrases thétiques de localisation temporelle (2a) ou dans les expressions le $N$ de $[+$ loc. temps] (2b) et la date du N (2c) :

\footnotetext{
${ }^{1}$ Les noms comme chanson, film et symphonie impliquent un rapport au temps et une forme de dynamicité, en cela qu'ils dénotent des entités structurées en phases et pouvant faire l'objet d'une exécution. Cependant, la dénotation d'entités spécifiques ne passe pas pour eux par la référence à un ancrage temporel : ils peuvent s'employer dans des SN spécifiques (e.g. un film de Howard Hawks) sans pour autant identifier leurs référents par leur exécution en un point donné du temps. Précisons que Flaux et Stosic (2011) considèrent aussi comme « noms d'idéalités » des noms iconiques (portrait, gravure), des noms d'entités langagières (mot, syntagme) et des noms d'objets mathématiques (nombre, théorème). Ces noms n'impliquent pas de relation directe au temps, et ne se comparent donc pas aux Nev.
} 

a. Il y a un concert à $20 h$.
b. la manifestation du mois dernier
c. la date de la réunion des délégués du personnel

Les Nev peuvent, d'autre part, dénoter des repères temporels, dans leur emploi avec les prépositions de temps (cf. Berthonneau 1989, Godard \& Jayez 1996, Haas 2009) :

$$
\text { durant le concert, après la manifestation, avant le festival de théâtre }
$$

Cette base sémantique commune n'exclut pas les disparités. Nous rappelons brièvement les principaux critères de différenciation des événements avant de nous interroger sur l'autonomie descriptive des Nev, relativement à ces différents critères.

\section{Typologie des événements}

Tels qu'ils sont décrits par les SN correspondants, les événements peuvent être de différents types. On distingue les événements selon qu'ils sont ou non duratifs, culminants, fortuits et spatiaux.

\subsection{Evénements duratifs / non duratifs}

Seuls certains Nev expriment la durée. D'autres dénotent des événements ponctuels, d'où leur incompatibilité avec les compléments de durée (de x temps) et le verbe durer (cf. Kiefer 1998, Haas et al. 2008) :
a. (une réunion / un concert / une vente aux enchères / un meeting électoral) de trois heures
b. *(un assassinat / une naissance / une démission / une découverte) de deux heures
a. (La conférence de presse / le dîner de gala / l'entraînement de l'équipe de France / l'assemblée générale des actionnaires) a duré trois heures.
b. *(Le crime / le décès / la collision entre les deux véhicules / la capitulation de l'armée ennemie) a duré deux heures.

Dans les cas où le Nev se présente (en synchronie) comme dérivé d'un verbe, la capacité d'exprimer ou non la durée est généralement héritée de la base verbale. Ainsi les Nev déverbaux ponctuels comme naissance et capitulation sont-ils dérivés de verbes d'achèvement.

\subsection{Evénements culminants / non culminants}

Les Nev se différencient par leurs propriétés de télicité. Seuls certains comportent dans leur structure événementielle un telos, i.e. un point culminant qui actualise l'événement dénoté. L'interruption des événements culminants empêche leur réalisation, comme le montre le « paradoxe imperfectif » existant pour les SV téliques (cf. Garey 1957, Kenny 1963, Dowty 1979). Adapté au domaine nominal, ce test conduit aux mêmes conclusions. Les événements non culminants dans (6) se distinguent des événements culminants dans (7) par le fait que leur interruption n'empêche pas d'inférer qu'ils se sont réalisés :

(6) a. La manifestation a été interrompue implique Ils ont manifesté.

b. La promenade a été interrompue implique Ils se sont promenés.

c. La discussion a été interrompue implique Ils ont discuté. 
a. L'accouchement a été interrompu n'implique pas Elle a accouché.

b. La vente du tableau a été interrompue n'implique pas Ils ont vendu le tableau.

c. La réparation du vélo a été interrompue n'implique pas $I l$ a réparé le vélo.

Les Nev dans (6) ont une structure événementielle homogène, en ce sens qu'elle ne comporte pas de point critique constituant à la fois l'objectif et le terme de l'événement décrit (cf. Haas \& Huyghe 2010).

\subsection{Evénements fortuits / non fortuits}

Les verbes supports événementiels permettent de distinguer différents Nev. En particulier, si tous sont compatibles avec avoir lieu, seuls certains se construisent avec se produire:

a. (Le séisme / une avalanche / le crash / le crime / un incident nucléaire / l'agression) s'est produit(e) en fin d'après-midi.

b. *(La réunion / la cérémonie / un concert / l'inauguration / le colloque / un entraînement) s'est produit(e) en fin d'après-midi.

Les Nev de (8a) dénotent, selon Gross (1996) et Gross \& Kiefer (1995), des événements « fortuits », c'est-à-dire des événements qui échappent au contrôle de certains au moins de leurs participants. Les événements dans (8b), au contraire, ne sont pas accidentels.

Les Nev incompatibles avec se produire dénotent tous des événements programmés. Ils se construisent avec les prédicats être prévu / être reporté [+ loc. temps] :

a. (Le mariage / le concert / l'examen / le vernissage / la rencontre) est prévu(e) le mois prochain.

b. (Le colloque / le procès / l'assemblée générale / le festival / le tournoi) est reporté(e) à l'an prochain. ${ }^{2}$

\subsection{Evénements spatiaux / non spatiaux}

Seuls certains Nev dénotent des événements physiques, i.e. des événements dont la réalisation dépend crucialement d'un ancrage spatial. Ces $\mathrm{N}$ peuvent figurer en position de cible dans le lieu $d u N(10)$ et peuvent dans certains cas apparaître en complément de lieu (11) (cf. Huyghe 2012b) :

a. Pierre est retourné sur le lieu (du crime / de la bataille / du tournage du documentaire / de l'avalanche / du crash du Concorde).

b. *Pierre est retourné sur le lieu (de l'augmentation du prix du gaz / du renouvellement des dirigeants / du démantèlement du consortium / de la prolongation du congé maternité / de l'ajustement budgétaire).

(11) a. Où était Sophie? - Elle était (au mariage de son collègue / à un colloque sur les adverbes / au concert de Kurt Wagner / à une réunion des alcooliques anonymes / au match France-Argentine).

\footnotetext{
${ }^{2}$ Un événement non programmé est fortuit, mais l'inverse n'est pas vrai. Il existe des Nev compatibles à la fois avec se produire et être prévu / être reporté (e.g. (L'attentat / l'assassinat / le cambriolage) (s'est produit / est prévu / est reporté à) la première semaine d'octobre). Ces Nev dénotent des événements prémédités, mais non contrôlés par l'ensemble de leurs participants. Il est ainsi possible de distinguer parmi les événements fortuits entre des événements intentionnels (i.e. à agent humain) et des événements non intentionnels (i.e. naturels).
} 
b. Où était Sophie ? - *Elle était (à une hausse de la criminalité / à une cessation de paiement / à un malentendu entre deux collègues / à son adhésion au club alpin / à un renversement des valeurs).

Les SN événementiels qui se combinent difficilement avec les expressions de localisation spatiale dénotent des événements mentaux (malentendu entre deux collègues), des changements idéologiques (renversement des valeurs), des procédures administratives (prolongation du congé maternité), des opérations financières (cessation de paiement), etc. L'existence des événements en question ne dépend pas prioritairement de l'ancrage dans le monde matériel.

\subsection{Combinaison de propriétés}

Les différentes propriétés événementielles présentées ci-dessus ne s'excluent pas mutuellement - seule l'absence de durée implique la culmination. Les événements décrits peuvent être par exemple :

(12) a. événement spatial, duratif, non culminant, non fortuit : concert

b. événement spatial, duratif, culminant, non fortuit : vente du tableau

c. événement spatial, non duratif, culminant, fortuit : crime

d. événement non spatial, duratif, non culminant, fortuit : hausse de la criminalité

e. événement non spatial, non duratif, culminant, non fortuit : adhésion au club alpin

f. événement non spatial, duratif, culminant, non fortuit : démantèlement $d u$ consortium

S'agissant des formes nominales impliquées, on observe que la catégorisation sémantique proposée doit parfois prendre en considération, non pas seulement le Nev, mais également certains compléments du N permettant de préciser l'événement dénoté. On peut donc se demander si les distinctions sémantiques proposées relèvent du lexique nominal ou de la construction syntagmatique.

\section{Nev autonomes et Nev dépendants}

Certains Nev ne suffisent pas à la description d'un événement : ils dépendent sémantiquement d'un complément permettant d'identifier l'événement dénoté. Ces Nev s'emploient difficilement seuls précédés d'un article indéfini dans des phrases existentielles. Ainsi les Nev de (14) contrastent-ils avec ceux de (13) :

(13) a. Il y a eu (un incendie / un cambriolage / une fête / un drame / un défilé / un orage / un décès / un bal / un enterrement / une tombola) dans le village hier.

b. (Un rassemblement / un concert / un accident / une kermesse / un drame / un tournage / un massacre / une manifestation / un suicide / un triathlon) a eu lieu dans le village hier.

(14) a. ?Il y a eu (une hausse / un glissement / une amélioration / une diffusion / un effondrement / une attribution / un bouclage / une accélération / une répartition / un ramassage) à Paris l'année dernière.

b. ?(Un accroissement / un ajustement / une vérification / un lancement / une participation / une dégradation / une pénalisation / une installation / un approvisionnement / une fabrication) a eu lieu à Paris l'année dernière. 
Les phrases (14) ne sont possibles qu'en cas d'ellipse du complément du $\mathrm{N}$, déjà connu ou reconstructible en contexte. La spécification d'un complément, comme dans (15), est nécessaire à l'identification d'un événement :

a. Il y a eu (une hausse des prix de l'immobilier / un glissement de terrain / une amélioration des conditions de travail) à Paris l'année dernière.

b. (Un accroissement des inégalités / un important ajustement budgétaire / une vérification du véhicule) a eu lieu à Paris l'année dernière.

Certains des $\mathrm{N}$ dans (13) peuvent régir un complément (e.g. le cambriolage de la villa, un rassemblement des nationalistes, le massacre des prisonniers), mais celui-ci n'est pas nécessaire à la dénotation d'un événement, ce qui constitue une différence cruciale avec les $\mathrm{N}$ dans (14).

Il existe donc deux types de Nev :

(i) des Nev sémantiquement incomplets, dépendants syntaxiquement d'un complément pour pouvoir dénoter un événement (e.g. augmentation),

(ii) des Nev sémantiquement complets, référentiellement autonomes (e.g. crime).

Une conséquence de l'autonomie des Nev du type (ii) est qu'on peut leur associer une classe référentielle homogène, comme c'est le cas pour les $\mathrm{N}$ « catégorématiques » standards, i.e. les $\mathrm{N}$ concrets qui dénotent des concepts autonomes (cf. Kleiber 1981 : 39). Contrairement à de nombreux Nev du type (i), les Nev du type (ii) peuvent s'employer dans des SN génériques sans complément :
a. Pierre (redoute / apprécie) (les incendies / les cambriolages / les fêtes / les réunions / les commémorations).
b. ??La population (redoute / apprécie) (les hausses / les améliorations / les destructions / les approvisionnements / les interruptions).

Aux Nev de (16b) ne correspondent pas de classes référentielles homogènes. Les emplois génériques de ces noms, comme leurs emplois indéfinis existentiels, nécessitent une expansion :

(17) La population (redoute / apprécie) (les hausses de prix / les améliorations des conditions de travail / les destructions d'emplois massives).

Dans la suite de ce travail, nous nous interrogeons sur la motivation morphologique ou syntaxique de la répartition entre $\mathrm{Nev}$ autonomes et dépendants, et sur un lien éventuel avec le type d'événement dénoté. Nous préciserons auparavant en quoi la distinction entre $\mathrm{Nev}$ autonomes et Nev dépendants diffère de celle, introduite par Grimshaw (1990) et exploitée dans de nombreux travaux sur les nominalisations, entre "simple event nominals » et « complex event nominals ».

\section{Nev autonomes / dépendants et « simple / complex event nominals »}

Grimshaw (1990) distingue deux types de nominalisations événementielles, suivant que le $\mathrm{N}$ a ou non une structure argumentale. Les nominalisations sont appelées " complex event nominals » [CEN] dans le premier cas et « simple event nominals » [SEN] dans le second. Selon cette conception, un Nev dépendant comme attribution est un CEN dans (18a), où il est doté d'une structure argumentale, et un Nev autonome comme cambriolage est un SEN dans (18b), où il est dépourvu de structure argumentale : 
a. L'attribution du marché public par cette commission doit être garante de neutralité. b. Il y a eu trois cambriolages dans le quartier la semaine dernière.

On peut se demander si la distinction entre $\mathrm{Nev}$ autonomes et Nev dépendants ne coïncide pas avec celle entre SEN et CEN.

\subsection{Arguments obligatoires / arguments spécifiés}

Une première différence entre Nev dépendants / autonomes et CEN / SEN tient à la présence d'une structure argumentale, en tant qu'association au N d'arguments « obligatoires ». La présence obligatoire d'arguments, caractéristique des CEN, n'implique pas leur spécification en contexte. Comme le note Grimshaw, «obligatory must mean the same for nouns as for verbs : capable of being obligatory but perhaps subject to lexical variation. After all, even direct objects of verbs are sometimes optional » $(1990: 49)$. Un argument « obligatoire » dans la structure peut faire l'objet d'une ellipse, i.e. d'une non-spécification en contexte. Or ce qui caractérise les Nev dépendants est la nécessité d'avoir un complément spécifié pour pouvoir référer. Dans le cas où ce complément correspond à un argument du N, l'existence d'une structure argumentale ne suffit pas à l'identification d'un événement : la connaissance spécifique des arguments en jeu est également requise. En cas d'anaphore, la dénotation des Nev dépendants repose, non pas strictement sur l'existence d'une structure argumentale associée au nom, mais sur la connaissance en contexte des arguments :

(19) (L'augmentation / le déclenchement / la divulgation / l'aggravation / le bouclage) a eu lieu le mois dernier.

On identifie les événements dénotés dans (19) à la condition, non pas uniquement que les Nev soient dotés d'arguments, mais que ces arguments soient précisés dans le contexte (e.g. l'augmentation du prix du gaz, le déclenchement de la procédure d'urgence, la divulgation des télégrammes diplomatiques, etc.). Les Nev dépendants manifestent donc une propriété de dépendance syntactico-sémantique différente de celle des CEN.

\subsection{Nev autonomes employés comme CEN}

Grimshaw (1990) observe que de nombreuses nominalisations peuvent s'employer dans les deux structures qu'elle décrit. Ainsi le nom cambriolage, employé comme SEN dans (18b), est un CEN dans (20), où il est pourvu d'une structure argumentale comprenant un agent introduit par par :

Le cambriolage de l'appartement par des malfrats cagoulés n'a pas encore été élucidé.

Grimshaw postule l'existence de changements de type SEN / CEN suivant l'emploi du N. Ces variations d'emploi ne relèvent toutefois pas de l'autonomie ou de la dépendance descriptive du N. Elles ne s'appuient pas nécessairement sur une polysémie lexicale. Envisager que ce soit le cas présenterait un risque d'amalgame entre le cas où un Nev autonome peut s'employer avec une structure argumentale sans changer de sens (e.g. trois cambriolages / le cambriolage de l'appartement par des malfrats cagoulés) et le cas où un $\mathrm{Nev}$ a deux acceptions, l'une autonome et l'autre dépendante, correspondant à l'absence ou à la présence d'une structure argumentale (e.g. une réception / la réception du colis). Ne pas dissocier l'autonomie / la dépendance sémantique d'un Nev de sa caractérisation comme SEN / CEN réduirait ces deux cas de figure à des doublons équivalents. La variation d'emploi dans le cas de réception, qui renvoie à une polysémie fondée sur l'autonomie ou la dépendance 
sémantique du Nev, ne serait pas distinguée de la variation d'emploi de cambriolage, qui est pourtant un Nev monosémique.

\subsection{Nev dépendants employés comme SEN}

Certains tests d'identification des SEN proposés par Grimshaw (1990) sont validés par les Nev dépendants. Par exemple, l'emploi au pluriel dans des SN comptables, notamment avec des adjectifs de fréquence (fréquent, répété, continuel, etc.), caractéristique des SEN et impossible pour les CEN, est permis pour de nombreux Nev dépendants :

(21) des destructions d'emplois répétées, des augmentations du prix du gaz continuelles, les renouvellements fréquents des dirigeants nationaux, de constantes améliorations des conditions de travail

Destruction, augmentation, renouvellement, amélioration présentent dans (21) les propriétés des SEN. Ils n'en restent pas moins référentiellement dépendants : ils nécessitent un complément pour donner une description suffisante des événements qu'ils dénotent.

Il ressort de nos observations que des $\mathrm{Nev}$ autonomes (e.g. cambriolage) et des $\mathrm{Nev}$ dépendants (e.g. augmentation) peuvent s'employer à la fois comme CEN et SEN. Le parallèle entre Nev autonomes / dépendants et SEN / CEN n'est donc pas établi.

\subsection{Nev dépendants dépourvus de structure argumentale}

Ajoutons que le complément nécessaire des Nev dépendants ne relève pas toujours d'une structure argumentale - comparable tout au moins à celles qui opèrent dans le domaine verbal. En effet, certains Nev dépendants ne sont pas des nominalisations :

\footnotetext{
?Il y a eu (une session / une phase / un trajet / une manche / une occurrence / une pénurie / un raid / une symbiose) à Paris ce matin.
}

Tous les Nev ne sont pas déverbaux (cf. Bittar 2010). Contrairement aux Nev dans (18)-(21), des $\mathrm{N}$ comme session, occurrence ou pénurie ne peuvent hériter de la structure argumentale d'un verbe source. La différence entre Nev dépendants et autonomes ne se réduit donc pas à la présence ou non d'une structure argumentale calquée sur le modèle verbal.

La question de savoir si les compléments des Nev dépendants non déverbaux ont le statut d'argument ne trouve pas de réponse aisée. On peut remarquer que dans certains cas au moins, le complément du $\mathrm{N}$ nécessaire à la description événementielle ne se voit pas assigner de rôle thématique semblable à ceux attribués par les prédicats verbaux. La dépendance sémantique des Nev non déverbaux peut tenir, par exemple, à une relation partitive, comme dans le cas de phase et manche. Les Nev dépendants n'ont donc pas tous un programme de complémentation comparable à celui des CEN.

D'une manière générale, la distinction entre SEN et CEN est élaborée pour rendre compte de deux types de constructions nominales, fondés sur des propriétés syntaxiques. La distinction entre Nev autonomes et dépendants est à visée lexicale. Elle est établie selon des propriétés sémantiques, avec des incidences syntaxiques. Pour Grimshaw (1990), seuls les CEN, parce qu'ils contiennent un noyau prédicat-arguments et expriment l'aspect, ont véritablement une interprétation événementielle. Grimshaw propose ainsi une définition syntaxique de l'événement, reposant sur la structure argumentale et aspectuelle, et correspondant aux propriétés de la catégorie verbale. Cette définition pose problème du point de vue sémantique, précisément parce qu'il existe des $\mathrm{N}$ dépourvus de structure argumentale, compatibles avec avoir lieu (take place ou occur en anglais) et communément considérés comme dénotant des événements (cf. Van de Velde 2006 : 107). La catégorie des SEN doit permettre de résoudre 
cette difficulté, mais le statut sémantique exact de ces N reste flou (Grimshaw 1990 : 58-59). Borer (2003) les regroupe avec les $\mathrm{N}$ résultatifs dans une classe qu'elle appelle les " referential nominals », par opposition aux " argument structure nominals » qui sont l'équivalent des CEN. Roy et Soare (2011), de leur côté, évoquent une possible distinction entre une événementialité "forte » et une événementialité "faible », correspondant respectivement aux CEN et aux SEN.

Il reste un point de convergence dans la multiplicité des approches, qui est le constat que certains $\mathrm{N}$ dénotant des événements sont, par leur autonomie syntaxique, plus proches que d'autres des noms résultatifs, i.e. de Nobj. On peut se demander s'ils s'en rapprochent également sémantiquement, par le type d'événement qu'ils décrivent.

\section{Répartition des Nev autonomes et dépendants}

Comment s'établit la répartition entre Nev autonomes et Nev dépendants ? Nous examinons successivement les particularités morphologiques et syntaxiques des Nev qui pourraient expliquer cette répartition.

\subsection{Construction morphologique}

On peut se demander si l'autonomie et la dépendance des Nev sont déterminées par leurs propriétés morphologiques, en particulier par leur caractère (non) construit. Les Nev autonomes et dépendants ne sont-ils pas respectivement des $\mathrm{N}$ simples et des $\mathrm{N}$ dérivés (construits à partir de bases verbales) ? ${ }^{3}$

Une majorité de Nev simples sont autonomes. Cependant, il existe aussi, comme nous l'avons vu dans (22), des Nev simples dépendants :

(23) a. Nev simples autonomes : crime, carnaval, grève, bal, procès, émeute, cérémonie, safari, séisme, kermesse, orage, miracle, etc.

b. Nev simples dépendants : phase, trajet, session, manche, occurrence, pénurie, éloge, symbiose, campagne, raid, tournoi, atelier, etc.

Par ailleurs, s'il est vrai que les Nev déverbaux sont souvent dépendants, de nombreux Nev déverbaux sont également autonomes :

(24) a. Nev déverbaux dépendants : augmentation, destruction, attribution, coupure, démantèlement, inflexion, vieillissement, diversification, tassement, fermeture, développement, durcissement, éclosion, élaboration, nivellement, bouclage, permutation, stabilisation, interdiction, élargissement, etc.

b. Nev déverbaux autonomes : cambriolage, mariage, exposition, tournage, réunion, manifestation, élection, agression, explosion, noyade, rassemblement, naissance, déménagement, bombardement, enterrement, commémoration, disparition, braderie, enlèvement, sabotage, etc.

Il n’y a donc pas de corrélation absolue entre la dépendance ou l'autonomie d'un Nev et le fait qu'il soit ou non un $\mathrm{N}$ construit.

\footnotetext{
${ }^{3}$ Les opérations de dérivation sont envisagées ici en synchronie. Un N est considéré comme déverbal s’il est lié morphologiquement à un verbe du français contemporain et s'il comporte un suffixe de nominalisation productif (e.g. -ion, -age, -ment). Abstraction est faite de l'éventuelle origine étymologique verbale des $\mathrm{N}$ « simples » dans (23), ou de l'éventuelle origine étymologique nominale des $\mathrm{N}$ « déverbaux » dans (24)-(27).
} 


\subsection{Héritage syntaxique}

Dans le cas des Nev déverbaux, l'autonomie ou la dépendance du N est-elle liée aux propriétés de transitivité du verbe de base ? Nous nous interrogeons plus particulièrement sur le rôle éventuel de l'(in)transitivité et de l'optionnalité des arguments du verbe source.

D'une part, la transitivité ou l'intransitivité du V ne motive pas la dépendance ou l'autonomie du $\mathrm{N}$ dérivé. Les $\mathrm{Nev}$ déverbaux, qu'ils soient autonomes ou dépendants, peuvent être construits à partir de verbes transitifs et intransitifs :

(25) a. Nev autonomes dérivés de V intransitifs : explosion < exploser, naissance < naître, manifestation $<$ manifester ${ }^{4}$, comparution $<$ comparaître, disparition $<$ disparaître, etc.

b. Nev dépendants dérivés de $\mathrm{V}$ intransitifs : éclosion < éclore, décélération < décélérer, cheminement $<$ cheminer, oscillation $<$ osciller, régression $<$ régresser, etc.

(26) a. Nev dépendants dérivés de V transitifs : destruction < détruire, attribution

$<$ attribuer, inflexion < infléchir, élaboration $<$ élaborer, renouvellement

$<$ renouveler, etc.

b. Nev autonomes dérivés de $\mathrm{V}$ transitifs : cambriolage < cambrioler, enterrement

$<$ enterrer, exposition < exposer, braderie < brader, tournage $<$ tourner, etc.

D'autre part, l'autonomie, pour les Nev dérivés de V transitifs, ne découle pas de l'optionnalité de l'argument interne du V source. Nev dépendants et autonomes peuvent être construits à partir de verbes dont l'objet est nécessairement exprimé. Ainsi les verbes dans (27), qui requièrent la spécification de leur objet direct, constituent la base morphologique de Nev dépendants (27a) ou autonomes (27b) :
a.?Pierre (détruit / infléchit / attribue / élabore / stabilise).
$>\mathrm{Nev}$ dépendants : destruction, inflexion, attribution, élaboration, stabilisation
b.? Pierre (cambriole / enterre / réunit / commémore / enlève).
$>\mathrm{Nev}$ autonomes : cambriolage, enterrement, réunion, commémoration, enlèvement

La capacité d'ellipse des arguments des Nev déverbaux n'est donc pas héritée de la base verbale.

\subsection{Restriction sémantique}

L'autonomie des Nev sémantiquement complets n'est due ni à leurs caractéristiques morphologiques, ni, dans le cas des $\mathrm{N}$ déverbaux, aux propriétés syntaxiques des verbes de base. Elle repose plutôt, dans ce dernier cas, sur une restriction sémantique (possible ou nécessaire) portant sur les arguments des $\mathrm{V}$ correspondants.

La contrainte de restriction peut être inhérente ou secondaire. Elle est inhérente lorsqu'un type d'argument par défaut est spécifié au niveau verbal, conditionnant l'autonomie du $\mathrm{N}$ dérivé. Tel est le cas pour des paires comme cambrioler $>$ cambriolage, marier $>$ mariage et assassiner $>$ assassinat. Les bases verbales ont un argument interne prototypique : [+habitation] pour cambrioler, [+humain] pour marier et assassiner. Cet argument type est repris implicitement et intégré dans la nominalisation, d'où l'autonomie référentielle du $\mathrm{Nev}$ dérivé.

\footnotetext{
${ }^{4}$ Manifester est considéré ici dans son emploi intransitif (e.g. Les agriculteurs ont manifesté à Paris ce matin vs L'enfant a manifesté sa colère en jetant ses jouets). De cet emploi dérive en effet le Nev autonome manifestation (Une manifestation a eu lieu à Paris ce matin vs Des manifestations de colère de l'enfant ont lieu chaque soir).
} 
La contrainte de restriction est secondaire lorsqu'un type d'argument, généralement [+humain], n'est spécifié qu'au niveau nominal. Tel est le cas pour des Nev comme rassemblement, disparition et enlèvement. Les verbes d'origine n'ont pas de prédilection pour un type d'argument interne particulier. La spécification opère uniquement au niveau nominal, où par défaut la nominalisation intègre un argument humain et suffit pour décrire un événement.

Ajoutons que le type sémantique du verbe de base pourrait contribuer à expliquer la répartition entre Nev déverbaux autonomes et dépendants. Par exemple, les Nev dérivés de $\mathrm{V}$ de création (fabrication, création, production) ou de modification de structure (éclatement, démantèlement, destructuration) semblent être des Nev dépendants. Le fait que les procès décrits dépendent essentiellement de leur objet pourrait expliquer que celui-ci fasse nécessairement l'objet d'une mention au niveau nominal.

\section{Autonomie des Nev et typologie des événements}

Les propriétés descriptives détaillées $§ 1$ sont marquées lexicalement pour les Nev autonomes. Mais toutes ne s'appliquent pas à l'ensemble des Nev dépendants. La description d'un événement spatial, en particulier, peut dépendre du complément du $\mathrm{N}$ :

(28) a. J'ignore quand a eu lieu (l'accumulation de ces déchets / cette apparition de la Vierge / l'installation du chapiteau / le redéploiement du bataillon).

b. J'ignore quand a eu lieu (l'accumulation de ces soupçons / l'apparition de cette méthode / l'installation de cette idéologie / le redéploiement des compétences).

(29) a. Nous nous rendons sur le lieu (de l'accumulation de ces déchets / de l'installation du chapiteau / de cette apparition de la Vierge / du redéploiement du bataillon).

b. ??Nous nous rendons sur le lieu (de l'accumulation de ces soupçons / de l'installation de cette idéologie / de l'apparition de cette méthode / du redéploiement des compétences).

En tant que noms dynamiques, les $\mathrm{N}$ accumulation, apparition, installation et redéploiement renvoient à des situations susceptibles d'être localisées dans le temps (28). Mais ils ne dénotent pas en soi des actions physiques ou non physiques. L'ancrage spatial de l'événement dénoté dépend du complément du $\mathrm{N}$, et notamment dans les constructions de la forme le $N_{l} d u$ $\mathrm{N}_{2}$, du type concret ou abstrait du $\mathrm{N}_{2}$. Accumulation, apparition, installation et redéploiement peuvent ainsi dénoter des événements permettant le repérage de lieux spécifiques, s'ils sont complétés par des $\mathrm{N}$ concrets (29a), et non abstraits (29b).

Des Nev comme accumulation ou installation se distinguent donc de Nev comme concert ou crime en cela qu'ils ne sont pas marqués lexicalement comme noms d'événements spatiaux ou non spatiaux. Il apparaît ici que la structure sémantique, i.e. l'ensemble des traits sémantiques pertinents, n'est pas la même pour tous les Nev.

On remarque par ailleurs que les événements non spatiaux sont majoritairement dénotés par des SN construits autour de Nev dépendants. Autrement dit, les Nev autonomes (concert, crime, cambriolage, fête, réunion, incendie, etc.) dénotent principalement des événements spatiaux. L'hypothèse suivante peut être formulée :

a. Nev autonomes $\rightarrow$ événements spatiaux

b. Nev dépendants $\rightarrow$ événements spatiaux / non spatiaux

L'autonomie ou la dépendance d'un Nev peut, suivant (30), conditionner le type d'événement dénoté. Si cette hypothèse est vérifiée, alors les Nev autonomes se rapprochent des Nobj, non 
seulement par leur autonomie syntaxique, mais aussi par leur signification concrète, c'est-àdire par leur capacité à dénoter des entités spatiales.

\section{Conclusion}

L'étiquetage lexical comme "nom d'événement » recouvre d'importantes disparités sémantiques, morphologiques et syntaxiques. Un point de différenciation sémantique fondamental est que certains $\mathrm{N}$, traditionnellement catégorisés comme $\mathrm{Nev}$, exigent un complément pour pouvoir dénoter des événements. Ces $\mathrm{N}$ décrivent en soi des actions, i.e. des situations dynamiques, mais pas à proprement parler des événements, i.e. des entités du monde. Les propriétés descriptives de ces Nev « dépendants » sont plus restreintes que celles des Nev « autonomes », qui comportent une description événementielle suffisante. Les traits sémantiques pertinents diffèrent pour les deux types de N. En effet, certains traits, comme ceux relevant de l'aspect lexical (culmination, durée), dépendent de l'actionnalité et sont impliqués par le trait de dynamicité, tandis que d'autres, comme la description d'entités spatiales, relèvent de l'événementialité au sens strict. La distinction entre Nev autonomes et dépendants constitue donc un préalable à une caractérisation sémantique fine des $\mathrm{Nev}$. Un usage scrupuleux de l'étiquette " $\mathrm{Nev}$ » pourrait exclure de la catégorie les $\mathrm{N}$ dépendants.

S'agissant de l'ontologie naïve véhiculée par la langue, on retiendra que, tels qu'ils sont dénotés par les $\mathrm{N}$ ou les $\mathrm{SN}$ correspondants, les événements peuvent être de différents types (duratif / ponctuel, fortuit / programmé, etc.). Une distinction majeure existe entre des événements spatio-temporels (e.g. un concert, une avalanche) et d'autres, essentiellement temporels (e.g. une augmentation du prix du gaz, une rupture idéologique). S’il existe dans le lexique un prototype événementiel, celui-ci correspond aux événements spatio-temporels. En effet, la classe des Nev est construite autour de $\mathrm{N}$ autonomes (e.g. crime, fête, avalanche, manifestation, concert). Les événements décrits sont physiques : ils sont ancrés à la fois dans le temps et dans l'espace.

\section{Références bibliographiques}

Balibar-Mrabti, Antoinette. 1990. Analyse d'adverbes en dans. Langue Française 86.

Berthonneau, Anne-Marie. 1989. Composantes linguistiques de la référence temporelle. Les compléments de temps, du lexique à l'énoncé. Thèse de doctorat d'état, Université de Paris 7.

Bittar, André. 2010. Construction d'un TimeBank du français : un corpus de référence annoté selon la norme ISO-TimeML. Thèse de Doctorat, Université Paris Diderot.

Borer, Hagit. 2003. Exo-skeletal vs. endo-skeletal explanations: syntactic projections and the lexicon. In The Nature of Explanation in Linguistic Theory, J. Moore \& M. Polinski (éds). Stanford : CSLI Publications.

Dal, Georgette \& Fiammetta Namer. 2010. Les noms en -ance/-ence du français : quel(s) patron(s) constructionnel(s)? In Actes en ligne du 2e Congrès Mondial de Linguistique Française, La Nouvelle Orléans, F. Neveu, V. Muni Toke, T. Klinger, J. Durand, L. Mondada \& S. Prévost (éds). Paris : ILF.

Dowty, David. 1979. Word Meaning and Montague Grammar. Dordrecht : D. Reidel Publishing Company.

Flaux, Nelly \& Danièle Van de Velde. 2000. Les noms en français : esquisse de classement. Paris : Ophrys.

Flaux, Nelly \& Dejan Stosic. 2011. Noms d'idéalités, prépositions et temporalité. In Temps, aspect et classes de mots : études théoriques et didactiques, E. Arjoca-Ieremia, C. Avezard-Roger, J. Goes, E. Moline \& A. Tihu (éds). Arras : Artois Presses Université. 
Gaatone, David. 1992. Les verbes événementiels et les dictionnaires : quelques observations. In Le mot, les mots, les bons mots. Hommage à Igor A. Mel'cuk à l'occasion de son soixantième annivervaire, A. Clas (éd.). Montréal : Presses de l'Université de Montréal.

Garey, Howard B. 1957. Verbal aspect in French. Language 33.

Godard, Danièle \& Jacques Jayez. 1996. Types nominaux et anaphores : le cas des objets et des événements. In Anaphores temporelles et (in-)coherence, W. De Mulder, L. Tasmowski-De Ryck, C. Vetters (éds), Cahiers Chronos 1, Amsterdam : Rodopi.

Grimshaw, Jane. 1990. Argument Structure. Cambridge Mass. : The MIT Press.

Gross, Gaston \& Ferenc Kiefer. 1995. La structure événementielle des substantifs, Folia Linguistica 29.

Gross, Gaston. 1996. Prédicats nominaux et compatibilité aspectuelle. Langages 121.

Haas, Pauline. 2009. Comment l'aspect vient aux noms. Les propriétés aspectuelles des noms à l'épreuve des restrictions de sélection imposées par certaines prépositions. Thèse de doctorat, Université de Lille 3.

Haas, Pauline ; Richard Huyghe \& Rafael Marín. 2008. Du verbe au nom : calques et décalages aspectuels. In Congrès Mondial de Linguistique Française - CMLF'08, J . Durand, B. Habert, B. Laks (éds), Paris : Institut de Linguistique Française.

Heyd, Sophie \& Marie-Laurence Knittel. 2009. Les noms d'activité parmi les noms abstraits : propriétés aspectuelles, distributionnelles et interprétatives, Lingvisticae Investigationes 32-1.

Huyghe, Richard. 2011. Evénements et objets dans l'espace et dans le temps : de l'ontologie à la langue. Cahiers de Lexicologie 99, 2011-2.

Huyghe, Richard. 2012a. Noms d'objets et noms d'événements : quelles frontières linguistiques? Scolia 26.

Huyghe, Richard. 2012b. Evénements et localisation spatiale : quand le lieu est un événement. In Langue, espace, cognition, B. Fagard \& D. Stosic (éds), Corela numéro thématique. http://corela.edel.univ-poitiers.fr/index.php?id=2786

Kenny, Anthony. 1963. Action, Emotion and Will. London: Routledge.

Kiefer, Ferenc. 1998. Les substantifs déverbaux événementiels, Langages 131.

Koehl, Aurore. 2009. Are French -ité suffixed nouns property nouns? In Selected Proceedings of the 6th Décembrettes, F. Montermini, G. Boyé, and J. Tseng (éds). Somerville, MA : Cascadilla Proceedings Project.

Martin, Fabienne. A paraître. Stage level and Individual level Readings of Dispositional Nouns. In Selected Proceedings of the 7th Décembrettes, N. Hathout, F. Montermini \& J. Tseng (éds).

Roy, Isabelle \& Elena Soare. 2011. Nominalizations : new insights and theoretical implications, Recherches Linguistiques de Vincennes 40.

Van de Velde, Danièle. 2006. Grammaire des événements. Villeneuve d'Ascq : Presses Universitaires du Septentrion. 